\title{
KLEINE BEITRÄGE ZUR GERMANISCHEN ALTERTUMSKUNDE.
}

\section{Skeáro á słé̃đi.}

IF. 17, 175 habe ich die redensart skera upp hergr $\mathrm{zu}$ erklären versucht. Das upp stammt aus wendungen wie skera upp bоð (herboð, bingboð). Hier vertritt es die präposition $a$ ' mit einem nomen, welches den gegenstand angeben würde, auf den das gebot geritzt wird. Man vergleiche sinnverwante ausdrücke: bað hann porgeir reisa bar upp ás ok skera á karlhofða; jarl lét rísta blóđorn á balki honum; hann tól við loorninu ok reist a rínar (Fritzner 3, 312a. 117 a). Ein *skera herboð (upp) a or war so gut wie unmöglich, weil eine solche abundanz des ausdrucks dem naiven sprechen, d.h. dem sprachlichen operieren mit geläufigen und als bekannt vorausgesetzten vorstellungen, immer fern liegt. $\mathrm{Zu}$ dem skera brauchte nur entweder die or oder das boð genannt zu werden; eins von beiden genügte.

Die abnormität liegt darin, dass statt *skera (upp) á heror vielmehr gesagt wird skera upp herpr. Rein sprachlich beschreiben wir diese verschiebung durch die proportion

skera herbod : skera herqu

(= láta fara orvarbod: láta fara herọr)

= skera upp herbor: $x$

$x=$ skera upp heror.

Eine ausreichende erklärung ist das jedoch noch nicht. Wie konnte skera (upp) ai heror durch die neubildung verdrängt werden? Einen teil der wahrleit erfassen wir vermutlich schon, wenn wir antworten: für die anschauung war der pfeil die hanptsache, nicht die inschrift; sein symbolischer wert überwog die bedeutung der zeichen; auch wo von herbod 
die rede war, schwebte zunächst der symbolische pfeil als solcher vor, nicht die zahlen oder runen, die er trug. Oder, etwas anders gewant: nicht beim schnitzen verweilte der gedanke, sondern bei der herumgeschickten $g r$, wie denn oft wenn nicht meistens - neben dem skera das láta fara nicht mehr besonders ausgedrückt wird. J)aher die vorliebe für den accusativus des resultats: skera upp heror, wie skera heror.

Aber vorliebe und ausschliesslicher gebrauch sind zweierlei. Wir dürfen aus dem nichtvorkommen des skera á or schliessen, dass den Isländern und Norwegern der schreibezeit diese wendung nicht mehr ohne weiteres verständlich gewesen wäre. Ohne zweifel haben wir es mit einer umdeutung zu tun. Man dachte bei dem grvarbod überhaupt nicht mehr an runen. Das unverstandene skera upp hat vielleicht gelegentlich ein aufschneiden, spalten suggeriert. Eine andere noterklärung bezeugt der vereinzelt vorkommende ausdruck skera upp p̈ing (Fritzner 3,313b); hier liegt association mit standa upp, reisa upp vor, es wird an die aufbrechende mannschaft gedacht wie in in nhd. aufbieten.

Das unverständnis wird damit zusammenhängen, dass skera nicht das eigentliche verbum für das runenschnitzen war. Doch der grund kann das widerum nicht sein. Es bliebe ja zu erklären, warum man nicht *rista á hergr sagte. Die wirkliche ursache ist zweifellos die, dass die alte praxis des orvarboð zur zeit unserer handschriften entweder längst ausgestorben war oder höchstens in formen fortlebte, die mit dem beritzten pfeil direct nichts zu tun hatten.

Vielleicht wird man sagen: dieses ergebnis war auf kürzerem wege zu gewinnen; es ist ja die einfachste folgerung von der welt. Gewiss, aber mir kam es darauf an, den survival-charakter unserer phrase recht'grell $\mathrm{zu}$ beleuchten. Um so bedeutsamer erscheint es nämlich, wenn wir an einer Eddastelle das ritzen des heeraufgebots noch als lebendige vorstellung belegen können.

Es handelt sich um Vsp. 20,5-12:

Urơ hétu eina, aðra Verðandi skáru á skíri - Skuld ena briðiu;

pær lqg logðu, pær líf kuru alda bqrnum - orlog seggia. 
KLEINE BEITRÄGE ZUR GERMAN. ALTERTUMSKUNDE. 1.461

Skáru á skídi fasst man mit recht als 'sie schnitzten (runen) in die holztafel' (Gering, Vollst. wb. 932). Auf diese tätigkeit der nornen bezieht man gewöhnlich v. 8--12. Der sinn müsste sein: sie schrieben das schicksal der menschen auf. Detter und Heinzel denken bei dem skíd an einen losstab, sagen aber nichts über den zusammenhang. Auch wenn wir den losstab aus dem spiel lassen, ist der zusammenhang nichts weniger als befriedigend. Jedenfalls haben wir $\mathrm{m}$. w. keine vergleichbare überlieferung. Wenn also schon der inhalt dazu ermutigt, eine bessere erklärung zu suchen, so verlangt die form gebieterisch danach. Was ist orlog seggia? Bugge bemerkt, seggia sei 3. plur. Aber ein präsens kann man hier nicht gelten lassen - abgesehen von der schiefen beziehung des seggia zu leggia und kiósa. Gering (Eddaübersetzung, Vollst. wb. 890) fasst seggia als gen. plur von seggr: 'der männer schicksal', und Boer hat sich dem angeschlossen (Zs. fdph. 36, 349, n. 1). Jedoch scheint die menschenanhäufung in der letzten langzeile kaum weniger bedenklich als die wechselnden tempora; Boer betont mit recht die dürftigkeit des inhalts, der sich bei dieser interpretation ergibt.

Die nächstliegende, auch durch die wortstellung empfohlene auffassung - diejenige, die Bugge ausschliessen wollte - halte ich zugleich für die richtige: seggia ist infinitiv. orlog seggia ist aufzufassen wie orlog drýgia im Wielandsliede: es heisst 'krieg anzusagen'. Das ist finale ergänzung zu skáru a' skídi, welches notwendig einer aufrundung bedarf: 'sie schnitzten runen auf dem stabe, krieg anzusagen'.

Grammatisch und stilistisch zunächst ist gegen diese deutung nichts einzuwenden. Diè verschlingung der sätze hat nicht ganz spärliche parallelen (Zs. fdph. 39, 296), auch in der Vsp. selbst, deren skaldischen neigungen sie gemäss ist (Vsp.12, wegen des nachklappenden schlussverses 16,8 ). In unserm fall geht die verkünstelung besonders weit. Nicht nur die langzeileneinheit wird syntaktisch ignoriert, auch die helminggrenze gilt nichts mehr. Das ist aber widerum auch anderswo belegbar ( $\mathrm{Akv}$., $\mathrm{Rp}$.). Es ist ein charakteristikum jüngerer denkmäler. An anderm orte gedenke ich auf diese erscheinungen in ihrem zusammenhange einzugehen. Unsere stelle hat in keiner hinsicht etwas unwahrscheinliches. 
Inhaltlich ist noch weniger anstoss zu nehmen. In str. 21 ist von dem ersten fólkvíg die rede. Seine entstehung durch die kriegsansage der nornen schildert eben str. 20. Als herrinnen des schlachtfeldes erscheinen letztere auch in den Hamðismál, wo die wölfe grey norna heissen. Dieselbe vorstellung dürfen wir in unsern versen $20,9-11$ suchen. Bei log hat der dichter vielleicht an aldrlag 'tod' gedacht. Dann erhalten wir einen sinnvollen gegensatz: 'sie setzten tod, erkiesten leben den menschenkindern', mit deutlicher beziehung auf die wechselfälle des krieges. Uebrigens hat man auch bislang schon die nornen an unserer stelle als kriegsanstifterinnen aufgefasst (Müllenhoff, DAk. 5, 95; Boer, Zs. fdph. 36, 348). Diese vermutung wịd bei unserer interpretation zur gewissheit.

Man kann die frage aufwerfen, ob der dichter wirklich das heeraufgebot gemeint oder ob ihm vielmehr eine fehdeansage vorgeschwebt hat. Der ausdruck skí muss wol letzten endes als mehrdeutig gelten. Bei weitem das wahrscheinlichste dürfte allerdings sein, dass er auf einen runenstab geht. Möglicherweise aber haben wir es mit einer uneigentlichen bezeichnung, wenn nicht des pfeiles, so des symbolischen schwertes oder kreuzes zu tun. Sollte letzteres gemeint sein, so spräche das für das aufgebot. Denn auf friesischem gebiet hat man ein schwertchen aus eibenholz gefunden mit einer runeninschrift, die Siebs (Pauls Grundr. 22, 521 f.) (k)eða bod$\not b(i n g)$ oder $(l) c d c e$ bodp(ing) oder (r)eda bodb(inga) liest. Dies schwertchen diente also sicher zum aufgebot. Seine form lässt vermuten, dass nicht das dingaufgebot, vielmehr das kriegsaufgebot seine ursprüngliche function war. Uebrigens bestätigt dieser fund den schluss, den ich IF. 17, 175 f. aus dem sprachlichen phänomen des skera upp gezogen habe: es hat wirklich beritzte symbole wie schwert und pfeil gegeben. Dass die schwerter auch im norden gebraucht wurden, ist an sich wahrscheinlich und scheint bestätigt zu werden durch das vorkommen von kreuzen in ähnlicher function (belege bei Fritzner 2, 353). Ein roh geschnitztes schwert ist von einem lateinischen kreuz nur unwesentlich verschieden; nichts ist natürlicher, als dass man solche friedlichen zwecken dienende schwerter in christlicher zeit kross zu nennen sich gewöhnte. - Diese kreuze sind wahrscheinlich von wikingen nach 
KLEINE BEI'TRÄGE ZUR GERMAN. ALTERTUMSKUNDE. 1.463

Schottland gebracht worden. Sie haben sich dort eingebürgert und wurden noch im 18. jh. angewant. Walter Scott hat sich darüber von einem manne berichten lassen, der bei den Stuartschen unruhen $1745 / 46$ selbst ein solches kreuz in umlauf gesetzt hatte (note zu The Lady of the Lake 3,1. Aus Scott hat der lexikograph Armstrong geschöpft, den Jacob Grimm, RA. 14,226 f. citiert). Auch das wort kefli ist ins englischschottische übergegangen (Sievers in Pauls Grundr. 12, 252; Wright, The English Dialect Dictionary I 458. III 412. 428).

Doch dies liegt ziemlich weit ab. Mit viel grösserer wahrscheinlichkeit haben wir, wie gesagt, in dem stíð einen einfachen runenstab zu sehen. Solche stäbe wurden ebenfalls zu ladungen gebraucht. Dafür haben wir, soweit ich sehe, zwei belegstellen. Die eine steht in der Niálssaga. Einige zeit vor der grossen brenna in Bergporshváll sieht ein mann einen gespenstischen reiter, der einen feuerbrand in der hand hält und eine strophe spricht mit der widerholten schlusszeile: svá ev um Flosa rád, sem fari kefli. Flosi ist der anführer der verbündeten, die den anschlag gegen Niáll vorbereiten. Man hat die zeile schon ganz richtig so aufgefasst, dass hier die schnelligkeit, mit der die gefahr heraufzieht, betont werden solle. Vigfusson denkt dabei an eine rollende walze, Fritzner an einen geschleuderten stab. Beides ist gleich willkürlich und pointelos und gewiss abzulehnen. kefli ist vielmehr hier wie meistens ein runenstab. fara erhält sein licht von der stehenden phrase láta fara grvarbod. Die schnelligkeit war mit dieser art des aufgebots eo ipso gegeben. Wie Scott berichtet, durchlief das 'feurige kreuz' im jahre 1745 eine strecke von 32 engl. meilen in drei stunden. ${ }^{1}$ ) Derartiges hat auch dem dichter unserer strophe vorgeschwebt. $\mathrm{Er}$ will sagen: der plan des Flosi gedeiht so schnell, er sammelt seine leute so geschwind, als bediente er sich eines kefli. Kein gedanke kann besser in den zusammenhang passen. Die verschwörer verständigten sich mündlich. Sie brauchten kein kefli, weil sie in der lage waren, sich zeit zu lassen. Aber als sie sich

1) v. Peucker, Das deutsche krieg:swesen 1, 234 ff. hat nachrichten des Caesar und Tacitus zusammengestellt über die schnelligkeit, mit der die Germauen bei römischen einfällen sich sammelten. Man darf für diese zeit schon eine ähnliche einrichtung wie das grvarbod mutmassen (s. u.). 
sammelten, sah es doch aus, als wären sie auf so eilige weise citiert worden. - Der zWeite zeuge tritt im 16. jh. auf. Olaus Magnus berichtet von einem baculus tripalmatus, der noch $\mathbf{z u}$ seiner zeit in Schweden die runde machte, sobald eine feindliche landung drohte (J.Grimm, RA.14,227). Dieser baculus und jenes $k$ efli sind jedenfalls nahe verwante des runenbrettes, das als vorstufe der böhmischen ladebrettchen zu vermuten ist. Auch das skíð der Voluspá. Aber kann letzteres nicht auch ein fehdezeichen sein? Der im Hunnenschlachtliede erwähnte herstafr wird ein rúnakefli gewesen sein. Doch diese alternative ist gewiss zu verwerfen. Wie alda bornum 20, 11 zeigt, müsste der dichter die nornen und menschen als gegner gedacht haben, und das ist einigermassen ungereimt. Auch liesse sich die offenbar vorhandene beziehung zu den zwischenstehenden sätzen log logðu, líf kuru kaūm aufrecht erhaltèn. Eine vorstellung wie die von den salcrinar, die Odin zwischen freunden hin und her trägt (HHu. 2, 34; Saxo 374: insidiosae legationis cura arctissimam regum concordiam labefectavit; vgl. Hárb. 24) wird durch den wortlaut unserer stelle ausgeschlossen.

So werden wir doch bei dem heeraufgebot bleiben. Es ist ein sinnreicher einfall des dichters, den krieg als ein herbod der nornen in die welt kommen zu lassen. Dieser einfall ișt aber zugleich für seine anschauungsweise, vielleicht auch für seine lebensstellung, lehrreich. Wir sehen hier, er betrachtet den krieg weder vom standpunkt des berufsmässigen kriegers noch vom standpunkt des historikers. Von beiden liesse sich erwarten, dass sie die wirklichen gründe und ziele des kriegführens - ruhmgier, herschsucht, habsucht, hass - irgendwie im poetischen bilde zeigen würden. Für unsern dichter ist der krieg ein schicksal, das von oben kommt. Er sieht ihn als runengeschnitztes herboð von gehöft. zu gehöft wạdern. Liegt die vermutung nicht nahe, dass dieser dichter selbst ein bauer war, der einmal oder öfter mit dem leiðangr ziehen musste?

$\mathrm{Zu}$ unserm ausgangspunkt zurückkehrend, stellen wir fest: das skera upp, das in der schreibezeit nicht mehr recht verstanden wurde, war dem verfasser von str. 20,5-12 der Vsp. noch geläufig. Er liefert uns eine nahe parallele $\mathrm{zu}$ dem $\mathrm{zu}$ erschliessenden *skera $a$ or. Hier verweilt nun wirklich die vorstellung auf der tätigkeit des skera. Die nornen treten 
RLEINE BEITRÄGE ZUR GERMAN. ALTERTUMSKUNDE. 1.465

runen ritzend auf; das aussenden der botschaft steht erst bevor (orlgg seggia, final). Es ist klar, dass die wahl des ausdrucks mit diesem stilistischen charakter der stelle zusammenhängt. Gewiss ist dem dichter auch die wendung skera upp herbod geläufig gewesen. Sie passte nur nicht in seinen zusammenhang, und daher sagte er skera á skíd, wobei freilich durchaus nicht feststeht, ob das stabende skid durch die phrase der umgangssprache vorgeschrieben war.

Es ist an sich wahrscheinlich, dass unsere stelle schon bei ihrer ersten aufzeichnung nicht mehr genau verstanden wurde. Dafür zeugt positiv die überlieferte textgestalt. Obgleich noch niemand $\mathrm{m}$. w. hier anstoss genommen hat, halte ich Urð hétu eina u.s.w. - das verbum im plural, die namen im acc. - für unursprünglich. Die schreiber haben sich wol die nornen selbst als subject gedacht, jedenfalls doch nicht die 'leute' (wie Rp. 39,8). Beides ist aber im grunde gleich unmöglich. Die nornen als subject sind nur gefolgert aus dem folgenden skárı á słídi, das man mit dem doppelton acc. Słculd ena priðiu als einheit fasste: 'sie verzeichneten als dritte die Skuld auf der tafel'. So haben denn auch die meisten neueren herausgeber diese zeile verstehen wollen; sie setzten hinter skíoi kein komma. Abgesehen von der inhaltlichen unmöglichkeit ist das nur eine consequente folgerung aus dem überlieferten wortlaut. Aber es scheint evident, dass Skuld durch ein misverständnis zum acc. gestempelt worden ist. Das ursprüngliche war:

$$
\begin{aligned}
& \text { Urðr hét ein, Qnnur Verðandi- } \\
& \text { skáru á skíri - Skuld en priðia u.s.w. }
\end{aligned}
$$

Bestätigend sagt die Rp.: Kundr hét einn $(41,9)$. Wie ich an anderer stelle zu zeigen hoffe, bestehen zwischen Rp. und Vsp. berührungen, die kaum zufall sein können. So hat gerade die citierte strophe in ihrer gliederung mit keiner stelle eine so auffallende ähnlichkeit wie mit Vsp. 12. Daher ist die vermutung berechtigt, dass Kundr hét einn auf Vsp. 20 beruht. Ferner kann man vergleichen Vkv. 2: ein . . onnur . en priðia. Es ist unverkennbar, dass durch unsere änderung die verse an klarheit und dadurch an ästhetischem wert erheblich gewinnen.

Nachtrag. In der verbindung orlog seggia haben wir eine der ursprünglichsten gebrauchsweisen des wortes orlgg. Diese bildung erklärt 
sich aus redensarten wie an. seggiask ór logum við e-n. Ein gegenstück dazu ist altd. urfehde schwören, das ebenfalls eine der ältesten anwendungen des wortes mhd. urvêhe(de) zeigt.

\section{Wgerm. schar.}

Bei vorstehender untersuchung scheint ein gewinn für die etymologie abzufallen.

Ueber schar 'cohors' bemerkt Kluge (Et. wb.), die bedeutung füge sich nicht zu scheren. Aehnlich Paul, Dt. wb. 373: 'abzutrennen ist schar in pflugschar, zu scheren, wovon auch das andere schar abgeleitet zu werden pflegt, aber ohne genügenden grund; die wurzel scheint vielmehr den sinn »ordnen«, »verteilen « gehabt z̃u haben'. Detter (Dt. wb., Lpz. 1897, s. 94) meint: 'vielleicht $\mathrm{zu}$ scheren "schneiden «, also eigentlich »abteilung «'. Diese auffassung hatte Heyne (Dt. wb. 8, 2170) als zweifellos richtig hingestellt. Er meinte demnach, dass hier das heer oder volk 'zerschnitten' gedacht werde, etwa wie man einen apfel vierteilt. Daran ist selbstverständlich nicht zu denken; etymologien wie diese hat Meringer mit recht gegeisselt. Wer die bedeutungen nicht besser zu vereinigen weiss, muss in der tat die verwantschaft bezweifeln.

Aber die bedeutungen kommen auf anderem wege zusammen. Wir haben gesehen, dass man einst den heerbann durch runenstäbe aufbot und dass diese art des aufgebots im an. mit hilfe des verbums skera sprachlich bezeichnet wurde. Dürften wir annehmen, dass beides, sitte und bezeichnung, einst gemeingermanisch war, so wäre der zusammenhang zwischen schar und scheren fast ohne rest aufgeklärt. Die schar konnte 'schnitt' oder 'ritzung' heissen, insofern es einer runenritzung bedurfte, um sie zur stelle zu bekommen. Haftete dem worte von hause aus der sinn 'abteilung' an, im gegensatz zum heerganzen, so wäre daran zu erinnern, dass die altgerm. heeresverfassung auf territorialer basis ruhte. Eine schar wäre dann das aufgebot eines bezirkes gewesen. 1) Es lässt sich ja annehmen, dass man bei einer mobilmachung in grösserem massstabe nicht nur ein aufgebot schnitzte, sondern etwa je eins für jeden bezirk. In Schottland pflegte der bote,

1) Vgl. die dingordnung des Södermannagesetzes: bupkafle en scal $i$ fiarbung hwarn (Noreen, Aschwed. lesebuch s. 16). 
der das 'feurige kreuz' trug, mündlich den sammelplatz anzugeben. Dasselbe berichtet Olaus Magnus. Vermutlich stand diese angabe früher auf dem holze selbst. Man kann sich wenigstens schwer vorstellen, was die runen sonst enthalten haben sollten. Alle andern vorschriften waren dem gegenüber unwesentlich, auch der zeitpunkt, der notgedrungen mit einem selbstverständlichen 'quam celerrime' abgetan wurde. Wenn nun jeder bezirk seinen sammelplatz hatte, so folgt schon hieraus, dass für jeden beżirk und damit für jede schar besondere runen geritzt werden mussten. Also schar $=$ runenschnitt.

Das wort ahd. skara u.s.w. 'schar' ist im nord. in dieser bedeutung nur als lehnwort vorhanden (in der piorekssaga skgr, in anderen jüngeren quellen skari, s. Cleasby-Vigf. und Fritzner). Einheimisches nordisches stegr hat von der grundbedeutung 'schnitt' aus verschiedene andere gebrauchsweisen entwickelt, von denen zwei hervorgehoben seien: skgr 'haupthaar', eig. wol 'schur', anfangs von der abgeschnittenen wolle des schafes ${ }^{1}$ ), dann, vielleicht ursprünglich spöttisch, ausschliesslich vom menschlichen haar, und die redensart skríða til skarar 'es zur entscheidung kommen lassen', über die unten gehandelt werden soll. Dass die bedeutung 'schar' nicht darunter ist, liegt daran, dass sich hierfür die ablautbildung skor (f.) festgesetzt hatte. Dieses skor ist zwar nur spärlich und in verengerter function belegt (evu fiogur hundvud $i$ skov, Fritzner, $3,367 \mathrm{~b}$ ), doch wird er durch das abgeleitete verbum skora (vgl. ahd. skarōn) 'herausfordern', 'citieren' gestützt. Wenn man sagte skora e-n til einvígis, á hólm, til leiðangrs, skora e-m hólm, so vergleicht sich damit das oben angezogene skera upp ping, das zwar mit skera upp heror u. dgl. associiert, aber nicht daraus entstanden war. Sollte nicht bei beiden verben ursprünglich das ritzen des aufgebots, bez. der herausforderung gemeint sein? skor bedeutet auch 'einschnitt', skora 'einschnitte machen'. Allerdings handelt es sich dabei nicht um runenzeichen. Aber es lässt sich in unsern quellen überhaupt kaum eine erinnerung an diesen gebrauch des wortes erwarten, da er auch bei skera nur noch an einer einzigen Eddastelle

1) Der t. t. für die schafschur ist in historischer zeit klippa (daneben rýja 'rupfen', Schönfeld, Isl. bauernhof s. 220 f.). Dies spricht nicht dagegen, dass man einmal skera ull so gut sagte wie skera mon, skera hár. 
direct belegt ist. Die besprochenen phrasen skera upp grvarbod, skera hergr u.s.w. sind ebenso erstarrte formeln wie skora e-m hólm. Letztere spiegelt aber genau die, oben vermutete angabe der örtlichkeit auf dem stabe. So wird man die annahme nicht zu kühn finden, dass skor 'haufe von 400 mann' in der tat aus der grundbedeutung 'ritzung', 'runenschnitt' zu erklären ist.

Diese erklärung dürfte jener andern vorzuziehen sein, die das aus dem nord. entlehnte engl. (a) score 'kerbholz', '20' an die hand gibt. Danach wäre die bedeutungsentwicklung diese gewesen: kerbe - kerbe als gedächtnishilfe (s. Fritzner s, v. skor 1) - eine bestimmte anzahl solcher kerben (etwa so viele, wie auf ein kerbholz von bestimmter länge gehen): 'kerbung' - diese anzahl als solche (20) - die quadratzahl davon (400) - truppe von 400 mann. Man müsste dann aber mindestens statuieren, dass die letzte specialisierung unter dem einflusse des deutschen lehnwortes skari, skgr erfolgt sei. Denn die semantische übereinstimmung mit dem verwanten westgerm. skara wird nicht zufall sein. Diese hypothese wäre bei der beschaffenheit der belege nicht a limine abzuweisen. Doch scheint die umgekehrte annahme vorzuziehen: ein älteres skor schar überhaupt' nahm die bedeutung 'schar von 400' an, weil man daneben ein gleichlautendes wort des sinnes ' 20 ' (vielleicht auch ' $20 \times 20$ ') hatte.

$\mathrm{Ob}$ diese erwägungen ein unśerer annahme günstiges vorurteil in betreff des wgerm. skara erzeugen können, lasse ich unerörtert. $\mathrm{Zu}$ berücksichtigen ist aber zweierlei: einmal ist es an sich höchst wahrscheinlich, dass die nordisch-friesische sitte des aufgebot-ritzens einst auch in Deutschland gegolten hat. Diese sitte war allem anschein nach im norden autochthon; andererseits ist ihr zusammenhang mit den von Meringer, IF. 16,114 besprochenen böhmischen brettern kaum zu leugnen. Was zweitens das verbum skera betrifft, so war sein gebrauch für das runenritzen im nordischen des 13. jh.'s, wie wir sahen, veraltet. Das begünstigt die vermutung, dass dieser gebrauch früher allgemeiner war. skeran dürfte der alte technische ausdruck für das schneiden der runen in weiches holz gewesen sein. Ein schnitzen, bei dem späne abfallen (s. die von Sievers in Pauls Grundr. 12, 251 citierte stelle der Gísla saga), wird 
KLEINE BEITRĀGE ZUR GERMAN. ALTERTUMSKUNDE. 2.469

durch dieses verbum besser bezeichnet als durch wrītan, das inschriften auf härterem material uns aufbewahrt haben.

Mir scheint, wir besitzen ein nicht zu verachtendes sprachliches zeugnis für die vorhistorische function von germ. skeran. Dabei gehe ich wider vom nordischen aus. Hier hat skera u. a. die bedeutung 'entscheiden'. Sie haftet in einigen festen formeln und an dem compositum skera ór (Fritzner 3, 312 b. 313a), wozu das abstractum órskurdr 'entscheidung' gehört (danach slíta ór, órslit in gleicher bedeutung). Was zunächst das ór angeht, so vergleicht Fritzner (2,915a) treffend wgerm. urteil. Die entsprechung lässt vermuten, dass die bildung alt ist, zumal die hier vorliegende function von $u z$ - isoliert $\mathrm{zu}$ stehen scheint. Wir dürfen demnach das einfache skera, soweit es mit skera ór synonym ist, auf urgerm. *usskeran zurückführen.

Eine der formeln, in denen unser nord. skera auftritt, ist láta skapat skera 'das schicksal entscheiden lassen'. Das erinnert an hd. bescheren, worüber J. Grimm, DWb. 1, $1563 \mathrm{ff}$. schön gehandelt hat: 'scheren, schar und bescheren gemahnen

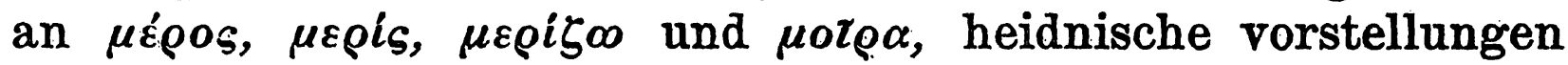
liegen ihnen allen im hintergrund'. Mhd. beschern wird besonders von der vorsehung und dem schicksal gebraucht (Mhd. wb. 2, 2, 155a; Lexer 1, 206). Aehnliches lässt sich im as. und ae. beobachten. Ettmüller (Lex. anglos.) führt an: scolde him

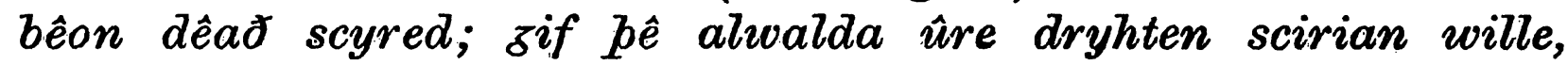
paet pî̀ rondwîzum rûmor môte froetwa doelan (entsprechende as. fälle s. u.). Nun handelt es sich hier ja allerdings um ein schwaches verbum, das als denominativum von schar zu fassen ist. Aber das ist nicht ausschlaggebend. Der gebrauch des wortes weist darauf hin, dass vermischung eines alten, wgerm. nicht mehr belegten skeran 'bestimmen' und eines nur wgerm. *skarjan 'einer schar zuteilen', 'wohin weisen' stattgefunden hat. Ad oculos demonstriert wird diese vermischung durch einen vergleich $z$ wischen dem ae. und dem an. Der an. formel skapa ok skera entspricht ae. scieppan ond scirian. Dabei besteht eine lehrreiche bedeutungsdifferenz. skapa ok skera ist eine rein tautologische doppelheit, ungefähr synonym mit jedem seiner glieder (s. die belege bei Fritzner 3, 284): 'entscheiden', 'die entscheidung haben'. Anders im ae. Dort fehlt Beiträge zur geschichte der deutschen sprachø, $\mathrm{X} \times \mathrm{XII1.}$ 
die tautologie, die formel wird z.t. gebraucht wie blosses scirian in dessen gewöhnlicher bedeutung 'wohin weisen'. Gen. 65: Sceôp $\not \hat{a}$ ond scyrede scyppend îre / oferhîdiz cyn engla of heofnum 'wies ... aus dem himmel'; d. menschen geschicke 95: Jod ... zeond middanzeard monna croftas / sceôp ond scyrede 'schuf ... und verteilte' (die belege aus Greins sprachschatz; auch 0 . Hoffmann, Reimformeln im wgerm. s. 70, hat nur diese beiden). Es ist wol klar, dass das ursprüngliche auf nordischer seite ist. Der ae. gebrauch hat seine voraussetzung in der verdrängung eines veralteten skeran durch scirian.

Dieselbe verdrängung beobachten wir, wenn scirian 'zählen' bedeutet. Das beruht auf skeran im sinne von 'einen einschnitt in ein kerbholz machen'.

Die grundbedeutung des swv. skerian liegt vor im Hildebrandsliede: dar man mith io skerita in sceotantero folk, im Heliand: thuo wurdun thar giskerida ... werôs te theru wahtu (5761 f.), ferner in mlat. scariti 'die aufgebotenen' (Waitz, Verf.gesch. 4,612); die ebenso ursprüngliche bedeutung 'aufstellen', an. fylkia zeigt Hel. 2848: hêt that gumono folk skerian enti skêðan. Von diesen ausgangspunkten lässt sich zwar begreifen, wenn skerian allgemein 'wohin weisen' ist - so ganz gewöhnlich im ae., ahd. (z. b. Petruslied v. 5), mhd. (Mhd. wb. 2, 2, 155 a. 156a) - aber schwerlich, wenn es 'bestimmēn', 'verhängen' - von irdischen gewalthabern und vom schicksal - bedeutet. Dies ist aber in zahlreichen belegen aus allen wgerm. dialekten der fall. Ich begnüge mich mit einigen altsächsischen: (God) skerida im thô te wîtea, that he ni mahta ênig word sprekan; hie was iu than te dôde giskerid; thena habdun sia giskerid te thiu (Hel. 164. 5446. 5647). Schon Schmeller (Bayr. wb. 22, 451) hat mit diesem skerian isl. skora verglichen. Noch besser hätte er skera herbeigezogen. Man denkt hier zunächst an skera upp bing 'ein thing berufen', dann aber an skera in der bedeutung 'entscheiden'. Diese ist perfective modificierung von 'bestimmen'. In letzterem sinne etwa wurde das urgerm. skeran gebraucht, das in wgerm. *skarjan aufgegangen ist.

Wie kam das verbum, das 'schneiden' bedeutete, zu diesem gebrauch? Nach dem, was oben ausgeführt wurde, liegt die antwort nicht fern. Die willensäusserungen, um die es sich hier handelt, wurden ursprünglich als runenritzungen gedacht. 
KUEINE BEITRÄGE ZUR GERMAN. ALTER'IUMSKUNDE. 2.471

Dabei sind in der theorie verschiedene fälle auseinander zu halten: der durch botschaft ausgehende befehl; die zauberrunen, durch die mensch oder gottheit das schicksal beeinflussen; die losstäbe.

Die nächste beziehung zu schar hat der erste fall. Von hier ist die vermischung der beiden verben ausgegangen. Wer eine schar sammelte (an. skera upp), hatte sie in der regel auch zu ordnen und zu führen (wgerm. skarjan). Beide tätigkeiten giengen ineinander über. Die folge war, dass die verben zunächst promiscue gebraucht wurden, *skarjan z. b. auch 'aufbieten' hiess (mlat. scarithi). Da aber von den concurrenten der eine (skeran) auch eine ganz andere function versah ('scheren', 'schneiden'), so musste er sich notwendig auf diese beschränken und dem *skarjan das feld überlassen. So begreift sich das fehlen der militärischen bedeutung bei wgerm. skeran und damit auch der semantische abstand zwischen scheren und schar.

Auf runenaberglauben geht zunächst bescheren zurück. Wie durch zaubersprüche geholfen und geschadet wurde, so auch durch runen, und das geheimnisvolle eingreifen der göttin geschah durch dasselbe medium.1) Zeugnisse dafür haben wir natürlich nur aus dem norden. Sie werden besprochen von Gering, Weissagung und zauber (Kieler rectoratsrede 1902) s. 20 f. (vgl. das. s. 8. 12). Man erregt durch runen liebe, bewirkt vergessenheit, krankheit und tod. Andererseits werden tote zum leben erweckt und krankheiten weggezaubert. Letzteres geschieht so, dass man die krankheitsrunen abschabt und durch heilrunen ersetzt.

Das gemeinwgerm. wort harmskara ist bisher nicht befriedigend erklärt. Am nächsten wird J. Grimm der wahrheit gekommen sein, wenn er RA. 24, 255 sagt: 'Scara ist auflage, herrenauflage, frohne, harmscara folglich was zur pein und qual (von der obrigkeit) auferlegt wird.' Er hat selbst später diese erklärung bezweifelt ${ }^{2}$ ), und es ist leicht zu erraten, warum. Zwischen harm 'leid' und skara 'herrengebot' ist eine beziehung nur gezwungen herzustellen. Das compositum könnte höchstens

1) Ueber die analogie zwischen zauberer und gottheit vgl. v. d. Leyen, Germ. abh. für H. Paul s. 51 ff.

2) S. die fussnote der herausgeber a. a. 0 . 
'leidvolle fronarbeit' bedeuten. Wenn es aber gemeinwgerm. für 'unglück' überhaupt gebraucht wird, so hat eine beziehung zu skara im angegebenen sinne nicht bestanden. Der zweite bestandteil von harmskara muss ungefähr 'schicksal' bedeutet haben. Es lässt sich aber beobachten, dass harmskara annähernd = harm ist. Entsprechendes haben wir in einer reihe an. composita mit dem zweiten bestandteil stafir:

$$
\begin{aligned}
& \text { blundstafir }=\text { blundr } \\
& \text { bolstafir }=\text { bol } \\
& \text { feiknstafir }=\text { feikn } \\
& \text { flærðarstafir }=\text { flærð. }
\end{aligned}
$$

Diese ausdrücke sind synonym mit harmskara; alle bezeichnen ein übel, das dem menschen widerfährt. Die zusammensetzung mit stafir zeigt, dass man sich das übel einmal als durch runenstäbe veranlasst gedacht hat. Mancher, der sich von bol betroffen fühlte, argwöhnte einen von einem widersacher, wenn nicht gar von einer gottheit geritzten 'burs', wie Skírnir der Gerðr mit dem purs und drei andern unglücksstäben droht. Wenn aber stäbe 'geschoren' wurden, so begreifen wir auch die harmschar: es ist eine 'harmbescherung'. Beleuchtet wird das noch durch eine altertümliche redensart, die uns die Niálssaga aufbewahrt: mikill harmr er at oss kveđinn, er vér skulum svá mikla úgofu saman eiga (Niála, Kaupmannahöfn 1875, c. 129, 96 f.). Der erklärende zusatz zeigt, dass der erste teil des satzes feste formel war. kveða at $e-m$ ist 'etw. über jem. verhängen', natürlich mittelst eines zauberliedes (kveða 'recitieren'). Setzen wir statt des liedes die verschwiegeneren runen ein, so erhalten wir harmr er at oss *skorinn, die voraussetzung für wgerm. harmskara. Wir dürfen es für zufall erklären, wenn dieses wort im nordischen selbst nicht nachgewiesen ist. - Beachtenswert ist das vorherschen des schädlichen zaubers. Die belege für runenzauber, soweit er nicht zum eigenen schutze dienen soll, gehören überwiegend auf diese seite. Hierher stellt sich auch harmskara, und so wird man das bairische b'schert 'mit unglück behaftet' ebenfalls aus heidnischem aberglauben herleiten dürfen.

Endlich die losstäbe. Dass man für das ritzen der losrunen auch einmal skeran gesagt hat, bezeugt, glaube ich, die an. phrase skríða til skarar 'es zur entscheidung kommen lassen'. skríða ist 
KI,EINE BEITRÄGE ZUR GERMAN. ALTER'TUMSKUNDE. 3. 473

gewöhnlich 'gleiten' oder 'kriechen'. Damit kommen wir hier nicht durch; Vigfussons 'to slide to the edge of the ice, i. e. to fight desperately' ist keine erklärung. Nun beruht ja der nordische gebrauch von skriða sichtlich auf später specialisierung. Wenn irgendwo, so kann sich die ältere, allgemeinere bedeutung in stabenden formeln gehalten haben. Eine solche formel ist slcriða $i$ skard 'einen schaden wider gut machen', eig. 'in die lücke treten'. skríða til skarar aber, aufs nächste vergleichbar der technischen phrase ganga til fréttar, ist ursprünglich 'zum losen schreiten', genauer 'zum ritzen der loszeichen schreiten'. Hier liegt derselbe secundäre gebrauch von schreiten vor, der heute im nhd. ganz gewöhnlich ist (zur abstimmung schreiten $\mathrm{u}$. dgl.).

Interessant ist die parallele der wörter los und kabel. Ersteres bedeutet von hause aus einen losstab, dann auch 'anteil' und 'schicksal'. Der anteil ist das, was man erlost, ebenso das schicksal. Mit los in der letzten bedeutung vergleicht sich unmittelbar hormskara, dessen zusammenhang mit dem runenstabe dadurch scharf beleuchtet wird. Wir beobachten hier, dass die vorstellungen 'los' und 'schicksalrune' ineinander übergehen. Der losstab, den man selbst geritzt, dem aber eine höhere macht beim loswerfen erst die wirkung gibt, und der stab, dessen runen durch sich selbst wirken, beide werden mit gleicher abergläubischer scheu betrachtet. - Was ndd. kabel betrifft, so erscheint beachtenswrert, dass es die bedeutungen 'reihenfolge' und 'anteil', besonders 'arbeitsanteil' mit schar gemein hat (DWb. 5, 7 f. 8, 2175). Die ursprüngliche bedeutung 'runenstab' zeigt an. kefli. Auch im norden galt einmal kefli für den losstab, wie das im östlichen und nördlichen England verbreitete, sichtlich entlehnte cavel (to cast cavels, Wright, Dialect Dict. 1,548) bezeugt. Aus diesem gebrauch erklären sich 'reihenfolge' und 'anteil'. Ebenso wird die entwicklung. bei schar gewesen' sein.

\section{Centum pagi.}

Caesar will über die Sueben gehört haben, dass sie 100 gaue hatten (Bell. Gall. 4, 1). Dasselbe berichtet Tacitus von den Semnonen, einem teilvolk der Sueben (Germ. 39). Die Hillevionen wohnten nach Plinius (Hist. nat. 4,96) in 500 gauen. 
Eine jüngere lateinische quelle weiss von centum pagi zwischen Rhein und ocean (Müllenhoff, DAk.4,461), die Gesta Francorum endlich ebenso von 100 gauen ' der Germanen oder Franken (Waitz, Verf.-gesch. 13, 223, n. 2).

Diese angaben wecken schon durch ihre stereotype einförmigkeit und die runden zahlen starke zweifel. Zwischen den berichten des Caesar und Tacitus besteht überdies ein widerspruch. Die verwirrung wächst noch, wenn wir einige weitere data herbeiziehen. Caesar lässt sich von den Treverern erzählen, pagos centum Sueborum ad ripas Rheni consedisse, qui Rhenum transire conarentur $(1,37)$. Das würde sich mit seiner späteren angabe über die Sueben nur vertragen, wenn das gesammtvolk gemeint sein könnte. Dies wird aber durch den wortlaut und den zusammenhang ausgeschlossen. Es hạdelt sich hier um leute, die dem Ariovist nachfolgen wollten, mit dem gewiss schon tausende von Sueben in Gallien waren; sie erleiden später durch die Ubier eine schwere schlappe $(1,54)$. Nicht minder auffallend ist eine stelle der Germ. c. 6. Hier ist die rede von der gemischten truppe, die vor der germanischen schlachtreihe kämpft: centeni ex singulis pagis. Man hat diesen bericht zu retten gesucht, so besonders Müllenhoff (DAk. 4,177f.), aber auch er kann nicht umhin. 'eine ungenauigkeit der darstellung und einen irrtum' anzunehmen (a.a. o. s. 176).

Ich glaube, dass der irrtum sich nicht auf diese stelle beschränkt. Die römischen berichte über das heerwesen der Germanen hängen offenbar alle mit einander zusammen. Wir haben hier eine tradition, an deren anfang ein verhängnisvolles misverständnis steht: die hundertschaft der germanischen oder gallischen gewährsmänner fasste man als. '100 gaue' auf. Das wird irgendwie damit zusammenhängen, dass diese einheiten nicht ausschliesslich militärischen charakter hatten, sondern auch einen gau (pagus) bedeuteten.

Diese auffassung ist nicht neu. Waitz (Verf.-gesch. 13, $220 \mathrm{f} .223 \mathrm{f}$.) erklärte Caesars bericht für eine fabel uud suchte hinter den centum pagi, ebenso hinter den centeni des Tacitus die misverstandene hundertschaft; er wante dies auch auf die Hillevionen an, bei denen er auf Áttundaland, Tíundaland verwies. Der historiker scheint aber mit seiner ansicht 
KLEINE BEITRÄGE ZUR GERMAN. ALTERTUMSKUNDE. 3.475

wenigstens bei germanisten nicht viel anklang gefunden $\mathrm{zu}$ haben. Bremer (Pauls Grundr. 32, 828) und Much (Dt. stammeskunde s. 136) glauben noch an die 500 gaue der Hillevionen, unter denen sie darum alle Skandinavier, bez. alle Ostskandinavier verstehen wollen. ${ }^{1}$ ) Hierin kann man diesen forschern schon deshalb nicht folgen, weil ein solcher gesammtname für jene zeit durchaus unglaubhaft ist. Wir haben allen grund anzunehmen, dass sich die Skandinavier damals noch nicht merklich von den südlicheren Germanen abhoben und sich diesen gegenüber nicht als einheit fühlten.

Neuerdings hat A. Bugge, offenbar unabhängig von Waitz, von neuem die vermutung ausgesprochen, die angabe des Plinius beruhe auf der misverstandenen nachricht, dass die Hillevionen in fünf hnndertschaften wohnten (Vesterl. Indflyd. S. 16).

Bei dieser sachlage scheint es mir nicht überflüssig, die frage noch einmal im zusammenhange zu behandeln. Ein so angesehenes werk wie der 4. band der Müllenhoffschen Altertumskunde vertritt noch immer ex professo die autorität der antiken schriftsteller, und andererseits hat Waitz, der die ansicht seiner gegner einer widerlegung für unwert lielt, sich ktirzer gefasst, als im interesse der sache lag, und ist dabei auch im verwerfen $\mathrm{zu}$ weit gegangen. Im folgenden kommt es mir nicht am wenigsten darauf an, den wahrheitskern klarzulegen, der namentlich in dem berichte Caesars steckt.

Kein Römer hat vom germanischen heerwesen klare vorstellungen gehabt. Es ist deshalb verfehlt, aus ihren dürftigen notizen grundlegende aufklärung schöpfen zu wollen. Vielmehr müssen wir von den einheimischen quellen ansgehen. Diese liefern uns die hundertschaft und stellen es durch ihre übereinstimmung ausser zweifel, dass namen und sache alt sind höchst wahrscheinlich älter als Caesar.

Wenn Caesar' zu hören glaubte, '100 gaue' der Sueben hätten sich am Rhein gelagert, sq waren einfach suebische

1) Bei Bremer finden sich allerding's daneben spuren der Waitzschen auffassung. Er erklärt c. pagis habitant durch 'nach hundertschaften organisiert' (s. 922) und spricht von der den Sueben und Semnen 'gemeinsamen eiurichtung der hundertschaften'. Much hilt sich folgerichtig auch hier an die quellen (s.110). 
hundertschaften gemeint; von ihrer zahl war keine rede. Ausgeschlossen ist es dabei nicht, dass der schriftsteller später gegen sein besseres wissen (vgl. 4, 1) die' '100 gaue' hat stehen lassen, damit man in Rom von ihnen läse; ein paar hundert Germanen schienen nicht genügend, um das vorgehen gegen Ariovist $\mathrm{zu}$ rechtfertigen.

Die spätere stelle $(4,1)$ lautet vollständig: $H i$ (Sıcbi) centum pagos habere dicuntur, ex quibus quotannis singula milia armatorum bellandi causa ex finibus educunt. Reliqui, qui domi mainserunt, se atque illos alunt; hi rursus invicem anno post in armis sunt, illi domi remanent. Sic neque agricultura nec ratio atque usus belli intermittitur. Danach hätte das jährliche aufgebot der Sueben sich auf 100000 mann belaufen, ihre gesammte mannschaft etwa auf das doppelte. Dies stimmt annähernd zu der gesammtżahl der Germanen, die Caesar in Gallien vorfand: es waren nach angabe des Aeduers Divitiacus rund $120000(1,31)$. Nun heisst Ariovist, der führer dieser scharen, auch rex Sueborum (DAk. 4, 29). Deshalb hat man sich berechtigt geglaubt, sein heer mit dem aufgebot der Sueben zu identificieren, und man hat die zahlen vollends gleich gemacht, indem man in den centum pagi ein grosshundert sah (Nitzsch und Müllenhoff, Zs. fda. 10, 552 f.). An dieser combination hat Müllenhoff bis an sein lebensende festgehalten (DAk. 4, 177 f.). Gleichwol fragt es sich, ob sie richtig ist. Ariovist liess dem Römer u. a. sagen, seine nie besiegten truppen seien 14 jahre lang unter kein dach gekommen $(1,36)$. Diese angabe ist sicher glaubwürdig in dem sinne, dass der stamm des heeres wirklich so lange auf der wanderung gewesen war. Bestätigend tritt der bericht des Divitiacus ein, nach dem zuerst - das heisst doch wol: vor mehreren jahren - 15000 über den Rhein gekommen waren, deren zahl allmählich auf 120000 angewachsen war. In dieses wetterharte kriegerleben setzte der germanische heerkönig seinen stolz, wie das noch ein jahrtausend später die nordischen sakonungar taten: pótti sá einn med fullu mega heita sakonungr., er hann svaf aldregi undir sótkum ási ok drakk aldregi at arins horni, Heimskr. 1, 57; Rognvaldr konungr var pá mestr hermadr i Vestrlondum; bat var priá vetr, or hann lá úti á herskipum svá at hann kom eigi undir sótkan raft, Flat.2,517. 
Lag also ein beträchtlicher teil von Ariovists heer schon jahrelang im felde, so kann es nicht mit dem suebischen aufgebot identisch sein. Denn dieses wechselte, wie Caesar ausdrücklich sagt, von jahr $z u$ jahr, und gerade das haben wir allen grund, ihm der hauptsache nach zu glauben (s. darüber weiter unten). Ferner ist es eine allzu gewagte behauptung, dass die Germanen, mit denen Caesar kämpfte, lauter Sueben waren. Caesars meinung ist dies jedenfalls nicht gewesen $(1,51)$. Und selbst wenn alle 120000 sich als Sueben betrachtet hätten, so wüssten wir immer aoch nicht, ob diese zahl nicht die frauen und kinder einschliesst (vgl. 1, 50,4-5. 51,3. 53, 4). Nicht gegen diese möglichkeit spricht es, wenn die Aeduer mit den 120000 gekämpft haben $(1,31)$, denn es scheint sich hier um eine reihe kleinerer gefechte $\mathrm{zu}$ handeln; von der zahl der streiter ist also keine rede.

Die gegenstandlosigkeit jener gleichsetzung erkennen wir vollends, wenn wir den bericht von dem auszuge der singula milia armatorum $(4,1)$ richtig würdigen. Wir tun gut, von den schematischen zahlen zunächst abzusehen. Was dann übrig bleibt, ist dies: alljährlich zieht etwa die hälfte der Sueben in den krieg, die andere hälfte bestellt zu hause den acker und sorgt für den unterhalt des ganzen volkes, und dies geht von jahr zu jahr um. Auch in dieser fassung steckt noch etwas von jenem schematismus, der dem römischen offizier und staatsmann nahe lag. Wir erkennen aber doch mit leichtigkeit den bedeutenden wahrheitskern, denn wesentlich dieselben zustände, die Caesar hier im auge hat, bestanden bei den nordischen Germanen noch tausend jahre später.

Wer in den skandinavischen quellen belesen ist, kennt die sitte, dass man im sommer auf die heerfahrt geht. Mit anbruch der wärmeren jahreszeit schwärmen die wikinge aus, um im herbst mit beute und ruhm heimzukehren. Sie nannten sich demgemäss sumarliðar. Solche sumarliðar waren es, die zu beginn des 9. jh.'s die fränkischen und englischen küsten heimsuchten. Erst allmählich gewöhǹten sich diese Normannen, im fremden lande regelmässig $z u$ überwintern. Daraus entstanden dann die grossen Normannenheere. ${ }^{1)}$ Daneben aler

1) Man vergleiche darüber Steenstrup, Normannerne I. II. 
hörten die sommerlichen züge aus der heimat nicht auf. Die Sachsenchronik erwähnt zufällig einen solchen zum jahre 871' $)$, wie es scheint, für die zeit um ostern. ${ }^{2}$ ) Auf den Orkneyinseln, dem letzten schlupfwinkel des wikingwesens, hielt sich diese lebensform bis ins: 12. jh. Besonders interessant ist der bericht der Orkneyinga saga über Sveinn Ásleifarson. Er vereinigte landbau und heerfahrt auf vorbildliche weise. Im frühjahr säte er mit seinen leuten das sommerkorn, heerte dann auf den Hebriden und in Irland bis zur erntezeit (was várvíking hiess) und segelte nach der ernte noch einmal auf haustviking, wovon er erst gegen ende september heimzukehren pflegte (Flat. 2, 512).

Die gewohnheit der sommerlichen raubzüge ist im norden sicher weit älter als die sogenannte wikingzeit. P. A. Munch hat bereits richtig erkannt, dass wir hier nichts anderes vor uns haben, als eine den landesverhältnissen entsprechende ausgestaltung uralter germanischer lebensformen. Latrocinia nullam habent infamiam, quae extra fines cuiusque civitatis fiunt, atque ea iuventutis exercendae ac desidiae minuendae causa fieri praedicant, sagt Caesar durchaus richtig $(6,23)$. Er schildert, wie solche unternehmungen zu stande kommen: ein princeps erklärt in der volksversammlung seine absicht auszuziehen und findet dann regelmässig ein zahlreiches gefolge (ebd.). Auch dies dürfen wir Caesar aufs wort glauben. Hier kommt das Germanentum, wie wir es aus den Isländergeschichten kennen, ungeschminkt zum vorschein. Caesar schreibt in der eile gegen sein besseres wissen, wenn er 6,35 sagt: Cogunt equitum duo milia Sugambri. Der beutezug, der im folgenden anschaulich geschildert wird, entsprang sicher auch der initiative irgend eines princeps, oder vielleicht mehrerer (vgl. Nasuam et Cimberium fratres 1,37). Es ist der einzige, von dem Caesar mit deutlichen worten berichtet, weil es der einzige ist, der über den Rhein gieng und den Römern gefährlich wurde. Zahlreiche solche räubereien und fehden aber spielten fortwährend zwischen den stämmen im innern Germanien. Wenn die Usipeter und Tencterer, der drang-

1) cuom micel sumor lida, zu ergänzen here? cyninz.

2) Es heisst unmittelbar darauf: ond bces ofer Eustron zefor ZEDered 
salierung durch die Sueben überdrüssig, schliesslich auswandern, die Ubier ihnen zins zahlen, so sind die 'kriege', die dem vorangiengen, keine grossen volkskriege gewesen, sondern einfälle einzelner häuptlinge. Bedeutsam ist das zeugnis Caesars 6, 23: Civitatatibus maxima laus est quam latissime circum se vastatis finibus solitudines habere... hoc se fore tutiores arbitrantur repentinae incursionis timore sublato (vgl. 4,3).

Wir sind nun vorbereitet, die vielberufene beschreibung der suebischen lebensweise zu verstehen. Schon aus der stelle selbst geht hervor, dass es sich um sommerliche auszüge handelt. Denn krieg wird nur in der guten jahreszeit geführt, und nur in dieser erfordert der ackerbau menschenkräfte. Also sitzt im winter die ganze bevölkerung daheim - soweit sie nicht im felde geblieben ist, müssen wir, mit Ariovists quattuordecim anni vor augen, hinzufügen. Im frühling zieht man aus. Educunt ist zu verstehen wie oben cogunt: nicht das volk beschliesst, sondern einzelne führer unternehmen. Stände nicht bellandi causa, sondern praedandi, so wäre niemand auf den gedanken gekommen, dass es sich hier um einen besondern fall handle, der sich aus dem kriegszustand mit Galliern und Römern erkläre (DAk. 4, 32 f. 370 f.). Diese altgermanischen völker waren immer oder nie im kriegszustand, je nach. dem, was man unter krieg versteht. bellare und praedari sind auf dieser culturstufe nahezu identisch. Was Caesar hier bellare nennt, ist ziemlich genau das nordische heria. In doppeltem sinne appelliert der princeps an seine volksgenossen: lasst uns reichtum erkämpfen (afla sér fiár) und ruhm suchen (leita sér sómðar). Beides kommt in dem oben citierten capitel $(6,23)$ deutlich genug zur geltung. Es ist auch an unserer stelle hinzuzudenken.

Caesar selbst hat bei dem bellare der Sueben nicht an ihren kriegszustand mit den Galliern gedacht, sondern an ihre lebensweise im allgemeinen, die sich ihm gerade unangenehm bemerkbar gemacht hatte. Nicht nur, weil Caesar im begriff war, die Sueben in ihrem eigenen lande aufzusuchen, schiebt er am anfange des vierten buches diese schilderung ein (so Müllenhoff, DAk. 4, 32). Der unmittelbare anlass war vielmehr der, dass die Sueben durch ihr bellare die Usipeter und Tencterer über den Rhein getrieben hatten. 
Es besteht eine deutliche beziehung zwischen dem exagitati bello $(4,1,2)$ und dem folgenden bellandi causa $(4,1,4)$. Ebenso läuft die schilderung in c. 3 wider auf die kriegerischen sitten des volkes aus, womit der schriftsteller über die Ubier zu den Usipetern und Tencterern zurückkehrt.

Darf man nun annehmen, dass es gerade die hälfte der streitbaren mannschaft war, die so jëdes jahr ihren führern folgte, und dass ausziehen und daheimbleiben jahr um jahr abwechselte? Mir scheint unbezweifelbar, dass die regelmässigkeit, die an eine gesetzliche vorschrift denken lässt, interpretatio romana ist. In wirklichkeit war hier ungleich mehr ungebundenheit, als die Römer sich träumen liessen. Man hat dem römischen feldherrn erzählt, wol die hälfte der Sueker treibe sich jeden sommer auf der heerfahrt herum, zu tausenden zögen sie aus, so unstät sei die art dieser leute (darauf geht auch das folgende: neque longius anno remanere uno in loco colendi causa licet). Vielleicht hat er ausserdem gehört, dass mancher nür ein jahr ums andere den acker bestelle, der not gehorchend, dazwischen aber seiner neigung folge und auf die ehrenvollere jagd nach vieh, waffen und goldenen ringen gehe. Aus solchen data etwa formte sich im geiste des Römers jenes schematische, unlebendige bild, das unserer zeit schon so viel kopfzerbrechen gemacht hat. Caesar hatte eben nichts vom ethnographen in sich, noch weniger als Tacitus, bei dem die liebe grösser gewesen sein dürfte als das verständnis. Es ist scherzhaft, in was für kostümen Gallier und Germanen in den commentarien auf= treten. Es erinnert an Horatius bei Corneille.

So haben wir den richtigen gesichtspunkt für die zahlen an unserer stelle gewonnen. Den zu grunde liegenden sachverhalt dürfen wir so formulieren: das suebische volk zerfällt in hundertschaften; aus jedem dieser gaue ziehen jährlich starke haufen, vielleicht die hälfte der mannschaft, auf die heerfahrt. - Man kann Müllenhoff (DAk. 4, 178) nicht zugeben, dass pagus hier eine tausendschaft bezeichne. Wenn der pagus 1000 krieger ins feld schickt und ebenso viele zurückbleiben, so ist seine ganze mannschaft 2000 köpfe stark!1)

1) Dies hat schon Waitz hervorgehoben (a.a. o. s. 224). 
KLEINE BEITRÄGE ZUR GERMAN. ALTER'TUMSKUNDE. 3. 481

Doch ich meine genügend gezeigt $\mathrm{zu}$ haben, dass dieses genaue rechnen überhaupt wertlos ist. Auf wie schwachen füssen das Müllenhoffsche system steht, erhellt schon daraus, dass centum, centeni bei ihm bald 100, bald 120 bedeuten müssen ') (Zs. fda. 10, 552: 'ein grosshundert aber als teilungszahl von 12000 genommen, ergibt die hundertschaft, 100 mann oder 50 reiter und 50 fussgänger', vgl. DAk. 4, 178). Das 'gespenst der suebischen verfassung', das Müllenhoff verjagt zu haben glaubte, hat gerade aus dem mystischen zahlenopfer, das er ihm brachte, neues leben getrunken.

Wir kommen zum Tacitus. Der fragliche passus in c. 6 तer Germania lautet: Definitur et numerus: centeni ex singulis pagis sunt, idque ipsum inter suos vocantur, et quod primo nimerus fuit, iam nomen et honor est. Besonderes gewicht legt Müllenhoff auf die zahl der reiterei des Ariovist, die nach Bell. Gall. 1, 486000 mann zu pferde und ebenso viele zu fuss enthielt. Diese 12000 bilden genau ein zehntel der gesammtmenge, wenn man diese nach $1,31=120000$ krieger rechnet. Dasselbe verhältnis hat nach Müllenhoff Tacitus im auge. Der gau (pagus), aus dem die 100 mann der gemischten truppe genommen sind, soll also eine tausendschaft sein.

Auch diese rechnung überzeugt nicht. Die tausendschaft ist bei den alten nirgends belegt; die zahl der krieger Ariovists steht nicht fest; und die runden zahlen, das glatte decimale verhältnis erregen um so stärkere bedenken, als es in der römischen geschichte fast die gleiche heeresverfassung gibt, die Caesar in der tat für Ariovists scharen angenommen zu haben scheint: das heer des Romulus soll aus 300 rittern (celeres) und 3000 mann fussvolk bestanden haben. Caesar sagt nicht, aus welcher quelle seine angabe der stärke der germanischen reiterei fliesst. Die vermutung liegt nahe, dass er sie geschätzt und die zahl auf grund jenes altrömischen verhältnisses, das ihm unbewusst als norm galt, fixiert hat. Ist das richtig, so kommt Caesar für die interpretation des Tacitus nicht in betracht. Denn das wird niemand annehmen wollen, dass letzterer tatsächlich das verhältnis 1 : 10 gemeint und es aus den eben erwähnten stellen bei Caesar abstrahiert

1) Vgl. Waitz a.a.o. s. 220, n. 2. 
482 NECKEL, KL. BEITRÄGE ZUR GERMAN. ALTERTUMSKUNDE. 3.

habe. Dazu sind die ausdrücke an der Germaniastelle viel zu unbestimmt. Ich glaube nicht, dass der schriftsteller hier überhaupt eine klare vorstellung ausdrückt. Vielmehr weist alles darauf hin, dass ein misverständnis im spiele ist. Hinter den centeni steckt nach allgemeiner annahme die hundertschaft. Tacitus weiss, dass das zahlwort im germanischen zu einem nomen geworden ist. Aber er hält diesen namen für einen ehrennamen, offenbar deshalb, weil er ihn nur in verbindung mit der vor der front aufgestellten gemischten truppe kennt. Das kann uns nicht auffallen, denn bei Tacitus zeigen sich auch sonst misverständnisse seiner germanischen quellen, und in Rom wusste man, wie gesagt, nichts von der germanischen hundertschaft. Der kern des berichtes ist: eine aus reitern und fussgängern gemischte hundertschaft pflegt vor der front zu stehen. Tacitus verstand 'hundertschaft' so wenig wie andere und machte daraus in erinnerung an Caesars 'je 1000 aus einem gau' sein centeni ex singulis pagis.

Auch seine nachricht über die Semnonen scheint direct mit Caesars Suebenstelle zusammenzuhängen. Man versteht diese und andere einzelheiten bei Tacitus am besten, wenn man mit Müllenhoff, DAk. 4,17 f. annimmt, er habe seinen vorgänger zwar gekannt und manche seiner angaben teilweise ungenau im gedächtnisse gehabt, aber nicht ihn benutzit. Tacitus war kein gelehrter schriftsteller. Seine werke wären für uns ungleich wertvoller, hätte er sich die mühe genommen, gewissenhaft seine quellen zu nennen, wie Ari. Er entschädigt uns dadurch, dass er die Germanen so merkwürdig findet.

BRESLAU, 10. juli 1907.

G. NECKEL. 